\title{
Features of Color Characteristics of Blood Plasma of Patients with Liver Cirrhosis in the Colorimetric System XYZ as Compared with Healthy People
}

\author{
Michail Dolomatov $^{1}$, Nikolay Kalashchenko ${ }^{2}$, Sergey Dezortsev ${ }^{1}$, Timur Araslanov ${ }^{2 *}$ \\ ${ }^{1}$ Department of Physics, Ufa State Academy of Economics and Service, Ufa, Russia; ${ }^{2}$ Bashkiria State Medical University, Ufa, \\ Russia. \\ Email: ${ }^{*}$ realitim@yandex.ru, dolomatov@gmail.com, Kalashchenko@mail.ru,jazz-n-blues@rambler.ru
}

Received January $8^{\text {th }}, 2012$; revised February $10^{\text {th }}, 2012$; accepted March $14^{\text {th }}, 2012$

\begin{abstract}
The authors determined a connection between change of functional state of human with liver cirrhosis and color characteristics of blood plasma. Features of color characteristics of blood plasma of patients with liver cirrhosis as compared with those of healthy people were determined by colorimetric method, based on absorption electromagnetic radiation in visible area. The average chromaticity coordinates in the XYZ system for patients with liver cirrhosis are $x=0.352 \pm$ $0.006, y=0.356 \pm 0.005$ as compared with healthy people chromaticity coordinates $x=0.32 \pm 0.001, y=0.32 \pm 0.002$.
\end{abstract}

Keywords: Absorption Spectrum; Plasma; Electronic Spectroscopy; Chromaticity Coordinates; Colorimetry

\section{Introduction}

The works in the field of studying integral spectral characteristics of human biological fluids showed the possibility of using electronic phenomenological spectroscopy for assessment of human health [1]. This method helped to find out that the integral characteristics of absorption of electromagnetic radiation (the integral oscillator power, the effective ionization potential and the effective affinity to electron) in the visible, ultraviolet and near-IR areas for aqueous solutions of plasma can serve as a criterion for evaluating functional state of human body [2]. Then the connection between the color characteristics of blood plasma in the XYZ colorimetric system and functional state of human body was determined $[3,4]$.

\section{Experimental Procedure}

A group of patients with compensated liver cirrhosis (46) and a group of donors (50) was selected for the investigation. Patients for the group were taken from the gastroenterology department of Republic Hospital using the certain criteria. The diagnosis of all patients was verified by biopsy. In addition, the diagnosis was confirmed by clinical data, results of biochemical blood tests and liver ultrasound data. The final diagnosis was made by experts from the gastroenterology department. The control group

${ }^{*}$ Corresponding author. consisted of donors from the blood transfusion department. All the donated blood was investigated by standard methods used in preparing of blood. Deviation was not found. Thus, the donors are considered to be the group of conditionally healthy people.

Spectra of blood plasma aqueous solutions were recorded on an electronic spectrophotometer in the wavelength range $380-780 \mathrm{~nm}$. All the measurements were performed for aqueous solutions of plasma with concentration $2.5 \%$ by volume.

Further, absorption coefficient $k\left(\lambda_{i}\right)$ was determined for each value of the wavelength $\lambda_{i}$ by spectrum of plasma. Spectral transmittance coefficients $\tau\left(\lambda_{i}\right)$ corresponding to the wavelength $\lambda_{i}$, were calculated using the Bouguer-Lambert-Beer principle :

$$
\tau\left(\lambda_{i}\right)=10^{-c \cdot k\left(\lambda_{i}\right) \cdot L}, \quad i=1,2, \cdots, n,
$$

where $\tau\left(\lambda_{i}\right)$-spectral transmittance coefficient of specific wavelength intervals $\lambda_{i}$ in accordance with intervals of spectrum decomposition, a dimensionless quantity;

$L$ - thickness of absorbing layer of solution, i.e. thickness of the cell, sm.

As you know, color characteristics depend on type of light source. The standard C source, CIE (Comission Internationale de l'Eclairage) was used as a source of visible light to determine color characteristics [5] phase of the day light (daytime diffused light of cloudy sky at noon in mid-latitudes) Color temperature Tc, K 6770. 
Color characteristics were calculated by standard methods of the CIE (radiation source $\mathrm{C}$ ) by the equations:

$$
\begin{aligned}
& \mathrm{X}=\int_{380}^{760} E(\lambda) \rho(\lambda) \bar{x}(\lambda) \mathrm{d} \lambda, \\
& \mathrm{Y}=\int_{380}^{760} E(\lambda) \rho(\lambda) \bar{y}(\lambda) \mathrm{d} \lambda \\
& \mathrm{Z}=\int_{380}^{760} E(\lambda) \rho(\lambda) \bar{z}(\lambda) \mathrm{d} \lambda
\end{aligned}
$$

where $\mathrm{X}, \mathrm{Y}, \mathrm{Z}$ - the color coordinates of red, green and blue lights, respectively, according to the colorimetric standard system CIE; $E(\lambda)$-function of spectral distribution of source; $\rho(\lambda)$-function of spectral reflec- tion or transmittance of blood plasma: $\bar{x}(\lambda), \bar{y}(\lambda)$, $\bar{z}(\lambda)$ - the average function of a standard observer.

The chromaticity coordinates were determined by the formula:

$$
x=\frac{\mathrm{X}}{\mathrm{X}+\mathrm{Y}+\mathrm{Z}}, y=\frac{\mathrm{Y}}{\mathrm{X}+\mathrm{Y}+\mathrm{Z}}
$$

\section{Experimental Results}

The differences between the spectra of donors and patients with liver cirrhosis are shown in Figure 1.

The color characteristics of the samples of blood plasma were calculated after processing spectra. The selective figures of the chromaticity coordinates of patients with liver cirrhosis and healthy people are shown in Table $\mathbf{1 .}$

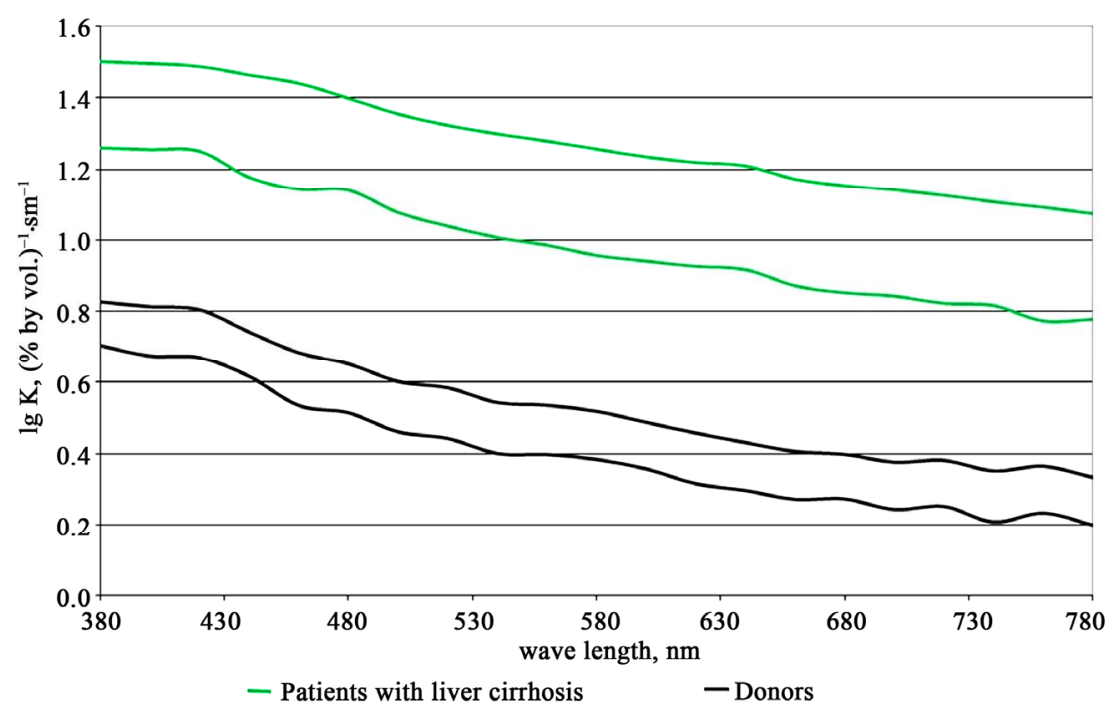

Figure 1. The confidence intervals of average spectra for patients with liver cirrhosis and donors in visible area.

Table 1. The chromaticity coordinates ( $x$ and $y$ ) of patients with liver cirrhosis and healthy people.

\begin{tabular}{ccccc}
\hline \multirow{2}{*}{ Samples of blood plasma } & \multicolumn{2}{c}{ Patients with liver cirrhosis } & \multicolumn{2}{c}{ Healthy people } \\
\cline { 2 - 5 } & $x$ & $y$ & $x$ & $y$ \\
\hline 1 & 0.348 & 0.350 & 0.322 & 0.324 \\
2 & 0.345 & 0.348 & 0.324 & 0.326 \\
3 & 0.341 & 0.343 & 0.318 & 0.315 \\
4 & 0.383 & 0.373 & 0.331 & 0.325 \\
5 & 0.348 & 0.357 & 0.321 & 0.315 \\
6 & 0.339 & 0.348 & 0.323 & 0.317 \\
7 & 0.360 & 0.368 & 0.315 & 0.316 \\
8 & 0.350 & 0.363 & 0.322 & 0.326 \\
9 & 0.372 & 0.375 & 0.330 & 0.331 \\
10 & 0.342 & 0.347 & 0.309 & 0.310 \\
11 & 0.344 & 0.346 & 0.313 & 0.311 \\
12 & 0.359 & 0.356 & 0.326 & 0.325 \\
13 & 0.345 & 0.347 & 0.317 & 0.318 \\
14 & 0.357 & 0.359 & 0.314 & 0.317 \\
15 & 0.353 & 0.360 & 0.319 & 0.325 \\
\hline
\end{tabular}


Table 2. The statistical characteristics of chromaticity coordinates for blood plasma of healthy individuals and patients with liver cirrhosis in the XYZ system.

\begin{tabular}{lcccc}
\hline \multirow{2}{*}{ Statistical indicators } & \multicolumn{2}{c}{ Patients with liver cirrhosis } & \multicolumn{2}{c}{ Healthy people } \\
\cline { 2 - 5 } & $x$ & $y$ & $x$ & $y$ \\
\hline Mean & 0.352 & 0.356 & 0.32 & 0.32 \\
Confidence interval & 0.006 & 0.005 & 0.001 & 0.002 \\
Dispersion, $\sigma$ & 0.02 & 0.02 & 0.005 & 0.008 \\
Error of mean & 0.003 & 0.002 & 0.001 & 0.001 \\
Variation coefficient & 5.62 & 4.80 & 1.6 & 2.6 \\
\hline
\end{tabular}

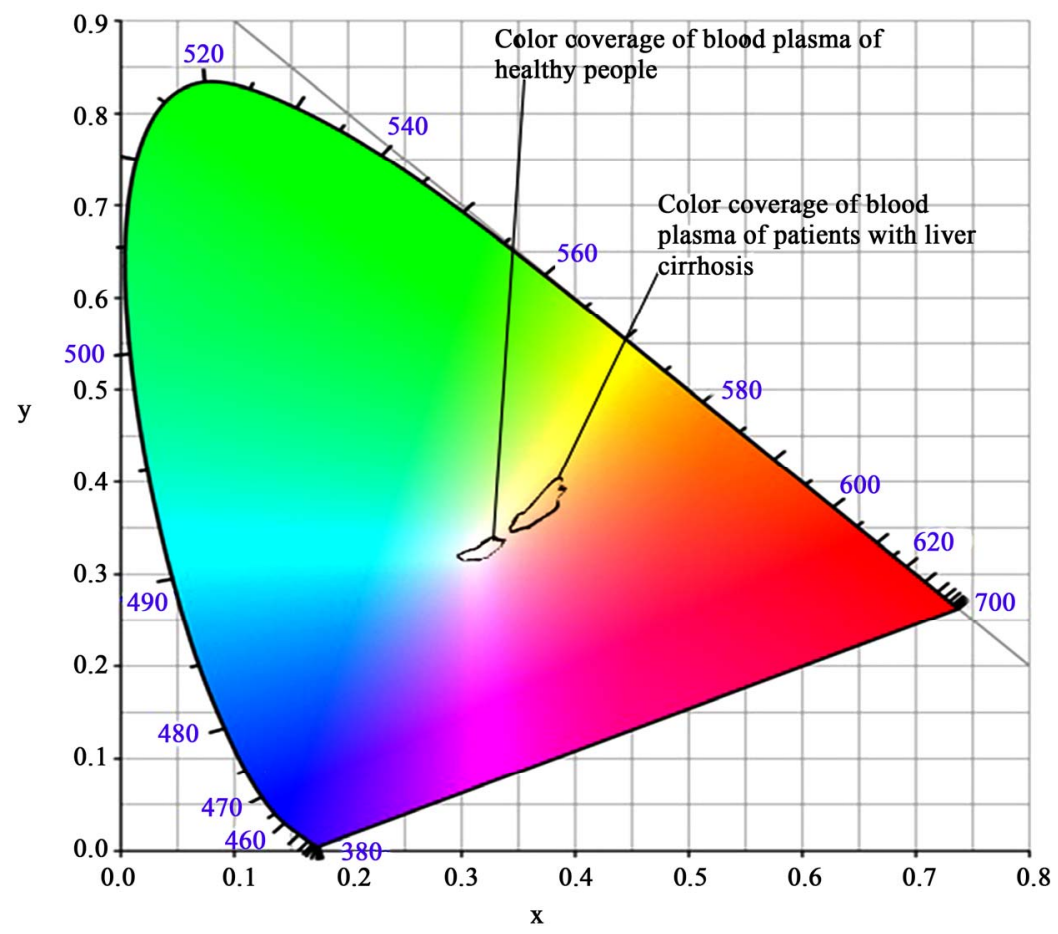

Figure 2. Blood plasma of patients with liver cirrhosis and healthy people on the chromaticity diagram of system XYZ (Source C).

Then the statistical analysis of the data was made. The basic statistics for all the investigated samples are shown in Table 2.

Totalities of samples have a distribution close to normal, and similar values of dispersion, therefore the t-test can be used to assess the reliability of the results. T of t-test for the chromaticity coordinate $x$ was 10.57 , for $y-12.9$. The critical value of $t$ for confidence probability $p=$ 0.999 is 3.5 , which is much smaller than the obtained results. Consequently, the differences between chromaticity coordinates of groups of patients and donors were statistically significant.

The differences between the color characteristics of blood plasma of patients with liver cirrhosis and healthy subjects are shown in Figure 2.
Colorimetric method established that a healthy person is characterized by the following indicators of chromaticity coordinates:

$$
x=0.32 \pm 0.001, y=0.32 \pm 0.002
$$

Patients with liver cirrhosis are characterized by the following color characteristics: $x=0.352 \pm 0.006 ; y=$ $0.356 \pm 0.005$.

\section{Discussion}

Having made the statistical processing the data we revealed that color characteristics of blood plasma of patients with liver cirrhosis differ from color characteristics of blood plasma of healthy people with a high degree of reliability. 


\section{Conclusion}

Investigation of human blood plasma by colorimetric methods can be used for express diagnosis of liver cirrhosis. A healthy person is characterized by the following indicators of chromaticity coordinates: $x=0.32 \pm 0.001$, $a=0.32 \pm 0.002$. Patients with cirrhosis of the liver are characterized by the following color characteristics: $x=$ $0.352 \pm 0.006 ; a=0.356 \pm 0.005$.

\section{REFERENCES}

[1] M. Yu. Dolomatov, N. V. Kalashchenko, S. V. Dezortsev, and T. R. Araslanov, "Spectroscopic Phenomenological Estimation of the Functional State of the Human Organism in Conditions of Environment," International Journal of Clinical Medicine, Vol. 2, No. 2, 2011, pp. 79-82
[2] N. V. Kalashchenko, M. Yu. Dolomatov and S.V. Dezortsev, "The Electronic Phenomenological Spectroscopy Blood of Human in Rate and Pathology. Theory and Practice Aspects," Inter, Moscow, 2010.

[3] N. W. Kalashchenko, M. Y. Dolomatov, S. V. Dezortsev, E. A. Popova and R. R. Kurmankaeva, "Normal and Pathological Color Characteristics of Human Blood Components," Journal of Applied Spectroscopy, Vol. 73, No. 2, 2006, pp. 245-250.

[4] L. A. Dolomatova, N. V. Kalashcenko, S. N. Dezorsev, and M. Y. Dolomatov, "Definition of Level of Persons Health on Color Features of the Biological Liquids in RGB Colorimetric System," Journal Biomedical Radio Electronics, Vol. 6, 2009, pp. 10-13.

[5] ISO/CIE 10526, "CIE Standard Illuminants for Colorimetry," International Organization for Standardization Geneva. 